\title{
Alterations of Uterine Blood Flow During the Follicular Phase in Patients With Recurrent Implantation Failure: A Doppler Ultrasonographic Study
}

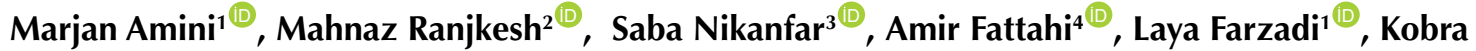 \\ Hamdi $^{*}$ (D)
}

\begin{abstract}
Objectives: The dynamics of blood flow in the endometrium plays a crucial role during the implantation process. This study aimed to assess the uterine perfusion during the follicular phase in patients with a history of recurrent implantation failure (RIF) and healthy fertile women using the transvaginal ultrasound color Doppler method.

Materials and Methods: To this end, 50 patients with RIF and 50 age-matched healthy fertile women were recruited in this casecontrol study. The transvaginal color Doppler ultrasonography was used to evaluate the pulsatility index (PI) and resistance index (RI) of the uterine, arcuate, and sub-endometrial arteries during the follicular phase in both groups.

Results: The RI and PI of both right and left uterine arteries were higher in the RIF group compared to the fertile women $(\mathrm{P}<0.05)$. Our results showed that the PI and RI of sub-endometrial blood flow and the RI of arcuate arteries were substantially higher in the group with a history of RIF in comparison with the control group. However, the PI of arcuate arteries was not significantly different between the groups.

Conclusions: Adequate uterine perfusion and sub-endometrial blood flow are necessary to achieve successful implantation and pregnancy since our results demonstrated the higher resistance of uterine and sub-endometrial arteries in patients with a history of RIF. Thus, the assessments of uterine perfusion indices during the follicular phase could be used as a non-invasive method in the evaluation of patients with RIF.

Keywords: Transvaginal Doppler ultrasound, Implantation failure, Uterine blood flow
\end{abstract}

\section{Introduction}

The endometrium plays a crucial role during the implantation and the subsequent supplying of nutrients to guarantee a successful pregnancy (1). Recurrent implantation failure (RIF) is among the best-defined reproductive defects referring to the pregnancy failure after the transfers of 1-2 embryos of high quality during at least three consecutive in vitro fertilization (IVF) cycles (2). Although there is a controversy regarding the precise definition of RIF, it can be defined by an unsuccessful transfer of 10 embryos in the cleavage stage or four blastocysts. One of the factors that can adversely affect the implantation is transferring the embryo to a non-receptive or dysfunctional endometrium $(3,4)$.

It has been reported that blood flow velocity in the uterine artery has an increasing trend between the early follicular phase and the time of implantation (5). Since the uterine perfusion regulates endometrium receptivity, impaired uterine blood supply may be involved in RIF in infertile patients undergoing the IVF procedure $(6,7)$.
In this regard, it has been demonstrated that an elevated pulsatility index (PI) of the uterine artery in women is related to the low chance of pregnancy in IVF/ICSI cycles $(8,9)$. In addition, Habara et al $(10)$ reported an association between the high impedance of uterine perfusion and recurrent pregnancy loss (RPL).

However, most of the studies on patients with RIF have investigated uterine perfusion during the luteal phase, and there is little information regarding the follicular phase. To identify the possible association between uterine perfusion during the follicular phase and implantation failure, the current study evaluated the blood flow of the uterine artery in the follicular phase of patients with a history of RIF and healthy fertile women using Doppler blood flow indexes.

\section{Materials and Methods}

Fifty women with a history of RIF and 50 age-matched normal fertile women were recruited in the present research. All women attended the Infertility Center of 


\section{Key Messages}

- The uterine blood flow of RIF patients is significantly altered in the follicular phase.

Al-Zahra Hospital of Tabriz, Iran. RIF was defined as a failure of pregnancy after the transfers of 1-2 high-quality embryo(s) during at least three consecutive IVF cycles. The decision about the type of protocol or doses of drugs was made by a gynecologist according to the ovarian response to the doses of the applied drugs during previous cycles. However, 150-400 IU/day of gonadotropins in combination with human menopausal gonadotropin (75-150 IU) were generally administered for 10-12 days during the gonadotropin-releasing hormone antagonist protocol. When the follicles reached a mean size of $>17$ $\mathrm{mm}$, ovulation was induced by 5000 or 10000 IU doses of human chorionic gonadotropin. After 36 hours, the cumulus-oocyte complexes were retrieved by the transvaginal ultrasound-guided puncture of the follicles. After checking the maturity of the denuded oocytes, metaphase II oocytes underwent the ICSI procedure. After the development of embryos, high-quality blastocysts (1-2 cases) based on Veeck and Zaninovic's criteria were selected for transfer (11). In this regard, the early formation of an expanded and eccentric cavity with a distinct layer of trophectoderm and inner cell mass was considered for the characterization of a good quality blastocyst. Women of the control group were ovulating females who experienced regular menstrual cycles and had no history of pregnancy failure. The inclusion criteria for all women were having 20-40 years of age with a body mass index of $<30 \mathrm{~kg} / \mathrm{m}^{2}$, no history of alcohol consumption or special diet, and being a nonsmoker. The patients with diabetes, dyslipidemia, uterine anomalies, uterine myomas, uterine adenomyosis, disorders of ovulation (including prolonged oligomenorrhea, premature ovarian failure, and hypothalamic amenorrhea), autoimmune diseases, hypertension, and coagulation disorders were excluded from the study. The participants were enrolled in the study after obtaining a signed informed consent form. All patients underwent Doppler transvaginal ultrasound to assess PI and RI of the right and left uterine, arcuate uterine, and sub-endometrial arteries.

\section{Transvaginal Doppler Ultrasound}

Transvaginal color Doppler ultrasonography was performed for both RIF patients and control women using the Samsung WS-80 ultrasound system (Samsung Medison Company Ltd, Seoul, South Korea) equipped with a 4 - to $8-\mathrm{MHz}$ transvaginal probe. The calculation software was used for measuring the PI and RI of the uterine arteries. The PI and RI were calculated from peaksystolic velocity (PSV) and end-diastolic velocity (EDV) using the following equations:
$P I=P S V-E D V /$ mean maximum flow velocity, $R I=P S V$ $E D V / P S V$

The alterations of uterine blood flow in the follicular phase were investigated for all participants.

\section{Statistical Analysis}

Due to time and financial constraints, the convenience sampling method was applied for the sample selection, and the sample size was calculated using the PASS 11.0 software (PASS, Kaysville, UT, USA). In this regard, beta and type I error were set to 0.20 ( $80 \%$ power) and 0.05 , respectively. Data are expressed as the mean \pm standard deviation (SD). After analyzing the data distribution by Kolmogorov-Smirnov test, variables were evaluated by the Student's $t$ test or the Mann-Whitney U test for data with a normal distribution or skewed ones, respectively. A $P$ value lower than 0.05 was considered statistically significant, and all statistical analyses were performed using SPSS software, version 16.0 (Chicago, IL, USA).

\section{Results}

This study recruited 50 RIF and 50 healthy women. The mean age of women in the RIF and control groups was $32.72 \pm 5.46$ and $31.84 \pm 4.21$ years, respectively $(P=0.342)$. Furthermore, the groups did not differ regarding the day of the follicular phase in which the ultrasonography was performed $(4.87 \pm 4.14$ in RIF women vs. $7.14 \pm 3.84$ in the control group, $P=0.103$ ), and the mean duration of infertility was $8.26 \pm 4.60$ years in the RIF group.

Embryo factors such as sperm and oocyte quality, chromosomal anomalies in parents, and the number and developmental stage of embryos can play a role in RIF. Furthermore, according to the guidelines of our fertility center, the number and developmental stage of embryos were approximately the same in most cases. Moreover, patients with uterine anomalies and autoimmune diseases were excluded from our study. However, this study did not assess all the possible reasons for RIF. Our results demonstrated that the total number of embryo transfer cycles and the total number of transferred embryos were $2.96 \pm 1.24$ and $10.36 \pm 8.21$, respectively.

Table 1 presents the means $( \pm S D)$ of PI and RI for uterine, arcuate, and sub-endometrial arteries in RIF patients and healthy women. The results of Doppler ultrasound examinations showed that the PI and RI of the right and left uterine arteries significantly increased in the RIF group in comparison with the control group. Moreover, the indices of pulsatility and resistance for sub-endometrial uterine arteries significantly elevated in women with a history of RIF compared to the control group $(P<0.001)$. The Doppler examination of the right and left uterine arteries and endometrial arteries in RIF women are shown in Figure 1, reflecting the increased resistance to blood flow while decreased perfusion in the uterine arteries. Based on our results, the RI of arcuate arteries 
Table 1. Mean $\pm \mathrm{SD}$ of $\mathrm{PI}$ and RI for Uterine, Arcuate, and Sub-endometrial Arteries in RIF and Control Groups

\begin{tabular}{lccc}
\hline \multirow{2}{*}{ Uterine Arteries } & $\begin{array}{c}\text { Study Group } \\
(\mathbf{n = 5 0})\end{array}$ & $\begin{array}{c}\text { Control Group } \\
(\mathbf{n = 5 0})\end{array}$ & \multirow{2}{*}{$\boldsymbol{P}$ Value } \\
\cline { 2 - 3 } & \multicolumn{2}{c}{ Mean $( \pm \mathbf{S D})$} & \\
\cline { 2 - 3 } Right uterine arteries & & & \\
PI & $2.96 \pm 0.61$ & $1.87 \pm 0.55$ & $<0.001^{*}$ \\
RI & $0.92 \pm 0.10$ & $0.73 \pm 0.14$ & $<0.001^{*}$ \\
Left uterine arteries & & & \\
PI & $3.05 \pm 0.69$ & $1.88 \pm 0.40$ & $<0.001^{*}$ \\
RI & $0.89 \pm 0.06$ & $0.74 \pm 0.14$ & $<0.001^{*}$ \\
Arcuate arteries & & & \\
PI & $1.87 \pm 0.61$ & $1.72 \pm 0.65$ & 0.229 \\
RI & $0.74 \pm 0.12$ & $0.65 \pm 0.17$ & $0.005^{*}$ \\
Sub-endometrial arteries & & & \\
PI & $1.478 \pm 0.87$ & $0.71 \pm 0.25$ & $0.001^{*}$ \\
RI & $0.67 \pm 0.20$ & $0.53 \pm 0.06$ & $<0.001^{*}$ \\
\hline
\end{tabular}

was significantly higher in the study group compared to the fertile women. However, the PI of arcuate arteries did not represent a statistically significant difference between the groups $(P=0.229)$.

\section{Discussion}

The dynamics of blood flow in reproductive tissues has an essential role in the endometrial growth and implantation, as well as the maturation of follicles and their subsequent conversion to corpus luteum (12-14). Given the important role of blood flow dynamics, alterations in blood supply (e.g., an increase in uterine artery resistance) may be responsible for diminished endometrial receptivity and thus RIF. Several studies have examined the role of the luteal phase in pregnancy loss $(10,15)$ while less attention has been paid to the importance of the follicular phase in these cases. However, it has been demonstrated that any defect in the follicular phase of the menstrual cycle could be associated with the poor quality of the oocyte and may compromise the quality of the embryo, and consequently, the pregnancy outcome (14). On the other hand, researchers rarely confirmed that the quality of oocyte can be a consequence of the vascularity and endocrinology of the follicular phase. Therefore, the present research aimed to investigate vascularity in the follicular phase in both RIF and healthy fertile women.

Our findings revealed vascularity variations in the uterus of women with RIF in comparison to the healthy women during the follicular phase. In this regard, higher resistance was found in the uterine arteries of patients with RIF compared to the control group. Based on our results, the RI and PI of the right and left uterine arteries and sub-endometrial arteries were higher in the study group compared to the controls. Our results are in line with the findings by Pattinaja et al, indicating a higher PI of uterine arteries in women with a history of at least two embryo transfers during IVF treatment compared to control women with normal reproduction (16). Furthermore,

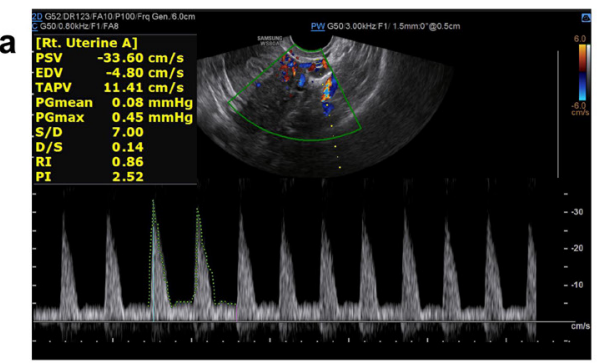

b

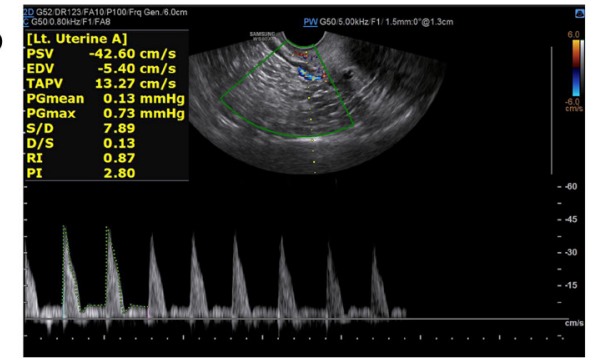

C

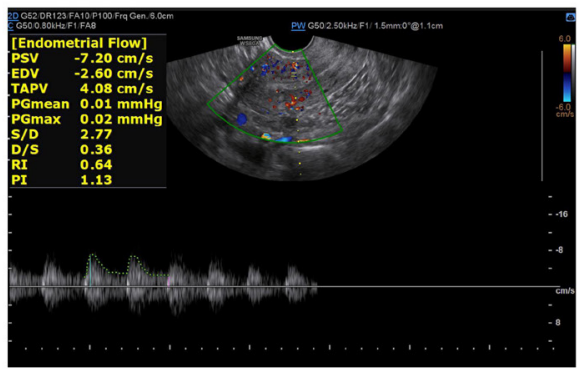

Figure 1. Doppler Examination of (a) Right Uterine Arteries, (b) Left Uterine Arteries, and (c) Endometrial Arteries in RIF Woman. Note. PI: Pulsatility index; RI: Resistance index. PI and RI were computed using equations PI $=($ PSV-EDV $) / T A P V$ and RI $=($ PSV-EDV $) /$ PSV. RIF: Recurrent implantation failure; Rt. uterine: Right uterine arteries; Lt. uterine: Left uterine arteries; PSV: Peak systolic velocity; EDV: End diastolic velocity; TAPV: Timed averaged peak velocity; PGmean: Mean pressure gradient; PGmax: Maximum pressure gradient; S/D: Systolic/diastolic ratio.

impaired uterine perfusion and decreased vascularity have been reported during the mid-luteal phase of the menstrual cycle in patients with RPL and recurrent miscarriage, respectively. Impaired uterine perfusion showed adverse effects on reproductive functions (17-20). It has been demonstrated that patients with RIF have a higher uterine artery PI in comparison with the examined infertile control cases during the mid-luteal phase before their first IVF trial (21). In this regard, individuals with successful implantation during the IVF procedure represented a low PI (22). Moreover, Abdel-Razik et al reported an increased $\mathrm{PI}$, along with the higher RI of the uterine arteries in patients with unexplained recurrent abortion (23). In contrast, some studies demonstrated that the mean of the PI of left and right uterine arteries in different days of the menstrual cycle was not significantly different between the women with pregnancy failure after embryo transfer and those with successful pregnancy $(24,25)$. Additionally, Prakash et al (14) reported no significant difference in the 
ultrasound measurements of patients with a history of pregnancy loss compared to the control group during the follicular phase, which is inconsistent with our results. It should be noted that in the above-mentioned study, luteal phase defects among approximately $30 \%$ of patients and a small sample size, especially for the control group could be the possible reasons for such a controversy. In addition, the assessment of Doppler indices between days 8 and 9 of the cycle could be another explanation for the discrepant results with the present study.

The results of the present study showed that the PI and RI of sub-endometrial arteries were higher in women with RIF compared to the controls. These findings conform to those of a previous study showing that RPL patients had an increased RI of sub-endometrial blood flow compared to normal controls (26). This is also consistent with reports of NG et al (27) regarding higher sub-endometrial vascularity in women with a successful live birth in comparison with those who experienced miscarriages. Two other studies linked implantation failure in IVF cycles with a decrease (28) or lack (29) of blood flow in the subendometrium. Furthermore, it has been represented that women with a successful IVF procedure had lower PI and RI of uterine and arcuate arteries compared to those with an unsuccessful IVF (30). Similarly, Yalti et al (31) found that women who conceived with intrauterine insemination had a lower uterine artery PI compared with those who did not conceive at all. This study was conducted during the follicular phase before human chorionic gonadotropin administration. Thus, blood flow impedance in uterine arteries may be an indicator of pregnancy success possibly due to the association of the vascularity of endometrium and sub-endometrium with the development of the placenta during pregnancy $(29,32)$.

The ischemia of the endometrium and subendometrium could be considered as one of the causative factors involved in RPL (26). In this regard, patients with miscarriage demonstrated significantly lower endometrial and sub-endometrial vascularity compared to pregnant women with live birth (27). However, due to the lack of a difference between the role of endometrial and subendometrial blood flow in getting pregnant, Chien et al proposed examining these two areas by color Doppler (28). Further studies on vascular changes, especially in pregnancy loss, may provide a broader understanding of the pathophysiology and etiology of these conditions.

Transvaginal Doppler ultrasound is a non-invasive method for measuring uterine vascularity (33) and can predict adverse pregnancy outcomes in high-risk women based on serum screening for pregnancy complications (34). Thus, finding markers to show the endometrial developmental defects and risk of pregnancy loss early in the follicular phase may provide the opportunity for better supporting the luteal phase or preventing unsuccessful embryo transfer (35). Therefore, evaluating the PI and RI of the uterus and arcuate and sub-endometrial arteries at the follicular phase could give useful information about endometrial receptivity. Moreover, the growing body of evidence regarding the critical role of sufficient uterine perfusion in pregnancy success makes it more important in the clinical approach. However, to validate our findings and define the mechanisms involved in the relationship between RIF and defective uterine artery blood flow in the follicular phase, further studies with a larger sample size are necessary.

\section{Conclusions}

In general, using transvaginal ultrasound examinations, it was revealed that the PI and RI of the right and left uterine arteries and sub-endometrial uterine arteries, along with the RI of the arcuate arteries were significantly higher in patients with a history of RIF compared to their control counterparts. These findings highlight the increased blood flow resistance and reduced uterine perfusion in RIF patients. In this respect, evaluating uterine perfusion early in the follicular phase may force physicians to better support the luteal phase or prevent embryo transfer. Finally, using Doppler sonography may be a useful noninvasive method for evaluating patients with implantation or pregnancy loss.

\section{Authors' Contribution}

Study conception or design: $\mathrm{KH}$; Acquisition of data: MR, MA, and LF; Analysis and interpretation of data: $\mathrm{SN}$; Drafting of the manuscript: $\mathrm{SN}$ and MA; Critical revisions: $\mathrm{AF}, \mathrm{KH}, \mathrm{MR}$, and LF.

Conflict of Interests

The authors declare that they have no conflict of interests.

\section{Ethical Issues}

The study received approval from the Ethics Committee of Tabriz University of Medical Sciences (IR.TBZMED.REC.1399.679).

\section{Financial Support}

This study was supported by Women's Reproductive Health Research Center, Department of Obstetrics and Gynecology, Faculty of Medicine, Tabriz University of Medical Sciences, Tabriz, Iran.

\section{Acknowledgments}

The authors would like to thank the staff of Al-Zahra Hospital for their help in conducting this research.

\section{References}

1. Ly KD, Aziz N, Safi J, Agarwal A. Evidence-based management of infertile couples with repeated implantation failure following IVF. Curr Womens Health Rev. 2010;6(3):200-18. doi:10.2174/157340410792007073

2. Simon A, Laufer N. Repeated implantation failure: clinical approach. Fertil Steril. 2012;97(5):1039-1043. doi:10.1016/j. fertnstert.2012.03.010

3. Valdes CT, Schutt A, Simon C. Implantation failure of endometrial origin: it is not pathology, but our failure to synchronize the developing embryo with a receptive endometrium. Fertil Steril. 2017;108(1):15-18. doi:10.1016/j.fertnstert.2017.05.033

4. Margalioth EJ, Ben-Chetrit A, Gal M, Eldar-Geva T. Investigation and treatment of repeated implantation failure following IVF-ET. Hum Reprod. 2006;21(12):3036-3043. doi:10.1093/humrep/del305

5. Steer CV, Campbell S, Pampiglione JS, Kingsland CR, Mason BA, Collins WP. Transvaginal colour flow imaging of the uterine arteries during the ovarian and menstrual cycles. Hum Reprod. 1990;5(4):391- 
395. doi:10.1093/oxfordjournals.humrep.a137109

6. Zegers-Hochschild F, Adamson GD, de Mouzon J, et al. The International Committee for Monitoring Assisted Reproductive Technology (ICMART) and the World Health Organization (WHO) revised glossary on ART terminology, 2009. Hum Reprod. 2009;24(11):2683-2687. doi:10.1093/humrep/dep343

7. Ng EH, Chan CC, Tang OS, Yeung WS, Ho PC. Factors affecting endometrial and subendometrial blood flow measured by threedimensional power Doppler ultrasound during IVF treatment. Hum Reprod. 2006;21(4):1062-1069. doi:10.1093/humrep/dei442

8. Tsai YC, Chang JC, Tai MJ, Kung FT, Yang LC, Chang SY. Relationship of uterine perfusion to outcome of intrauterine insemination. J Ultrasound Med. 1996;15(9):633-636. doi:10.7863/jum.1996.15.9.633

9. Cacciatore B, Simberg N, Fusaro P, Tiitinen A. Transvaginal Doppler study of uterine artery blood flow in in vitro fertilization-embryo transfer cycles. Fertil Steril. 1996;66(1):130-134. doi:10.1016/s00150282(16)58400-3

10. Habara T, Nakatsuka M, Konishi H, Asagiri K, Noguchi S, Kudo T. Elevated blood flow resistance in uterine arteries of women with unexplained recurrent pregnancy loss. Hum Reprod. 2002;17(1):190194. doi:10.1093/humrep/17.1.190

11. Veeck LL, Zaninovic N. An Atlas of Human Blastocysts. New York: Parthenon Pub. Group; 2003:286.

12. Nardo LG. Vascular endothelial growth factor expression in the endometrium during the menstrual cycle, implantation window and early pregnancy. Curr Opin Obstet Gynecol. 2005;17(4):419-423. doi:10.1097/01.gco.0000175362.12470.e0

13. Demir R, Kayisli UA, Cayli S, Huppertz B. Sequential steps during vasculogenesis and angiogenesis in the very early human placenta. Placenta. 2006;27(6-7):535-539. doi:10.1016/j.placenta.2005.05.011

14. Prakash A, Li TC, Laird S, Nargund G, Ledger WL. Absence of follicular phase defect in women with recurrent miscarriage. Fertil Steril. 2006;85(6):1784-1790. doi:10.1016/j.fertnstert.2005.11.045

15. Shimada S, Kato EH, Morikawa M, et al. No difference in natural killer or natural killer T-cell population, but aberrant T-helper cell population in the endometrium of women with repeated miscarriage. Hum Reprod. 2004;19(4):1018-1024. doi:10.1093/humrep/deh159

16. Pattinaja DA, Spaanderman ME, Ghossein-Doha C, van Golde RJ. Repeated implantation failure relates to circulatory abnormalities. Fertil Steril. 2015;104(3):e338. doi:10.1016/j.fertnstert.2015.07.1053

17. Yang W, Wu Z, Yu M, et al. Characteristics of midluteal phase uterine artery hemodynamics in patients with recurrent pregnancy loss. J Obstet Gynaecol Res. 2019;45(7):1230-1235. doi:10.1111/jog.13944

18. Tan SY, Hang F, Purvarshi G, Li MQ, Meng DH, Huang LL. Decreased endometrial vascularity and receptivity in unexplained recurrent miscarriage patients during midluteal and early pregnancy phases. Taiwan J Obstet Gynecol. 2015;54(5):522-526. doi:10.1016/j. tjog.2014.10.008

19. Wahab HA, El-Din DS, Zain E, Abdelgany M, Youssef MAFM. Uterine artery Doppler and subendometrial blood flow in patients with unexplained recurrent miscarriage. Middle East Fertil Soc J. 2011;16(3):209-214. doi:10.1016/j.mefs.2011.04.001

20. Chen L, Quan S, Li H, Chen C, Xing F, Yu Y. A comparison of endometrial and subendometrial vascularity assessed by threedimensional ultrasonography and power Doppler angiography between healthy fertile women and women with unexplained primary recurrent miscarriage. Fertil Steril. 2011;95(3):1127-1129. doi:10.1016/j. fertnstert.2010.09.034

21. Eid ME, Taye AM. Impaired uterine perfusion in infertile women with repeated IVF-implantation failure as detected by uterine artery color Doppler study. Fertil Steril. 2009;92(3):S118. doi:10.1016/j. fertnstert.2009.07.1126

22. Steer CV, Campbell S, Tan SL, et al. The use of transvaginal color flow imaging after in vitro fertilization to identify optimum uterine conditions before embryo transfer. Fertil Steril. 1992;57(2):372-376. doi:10.1016/s0015-0282(16)54848-1

23. Abdel-Razik M, El-Berry S, Mostafa A. The effects of nitric oxide donors on uterine artery and sub-endometrial blood flow in patients with unexplained recurrent abortion. J Reprod Infertil. 2014;15(3):142-146.

24. Hoozemans DA, Schats R, Lambalk NB, Homburg R, Hompes PG. Serial uterine artery Doppler velocity parameters and human uterine receptivity in IVF/ICSI cycles. Ultrasound Obstet Gynecol. 2008;31(4):432-438. doi:10.1002/uog.5179

25. Prasad S, Goyal R, Kumar $Y$, et al. The relationship between uterine artery two-dimensional color Doppler measurement and pregnancy outcome: a prospective observational study. J Reprod Infertil. 2017;18(2):251-256.

26. Mansour GM, Hussein SH, Abd El Hady RM, et al. Uterine artery flow velocity waveform (FVW) type and subednometrial vascularity in recurrent pregnancy loss. J Matern Fetal Neonatal Med. 2020;33(4):527532. doi:10.1080/14767058.2018.1495190

27. Ng EH, Chan CC, Tang OS, Yeung WS, Ho PC. Endometrial and subendometrial vascularity is higher in pregnant patients with livebirth following ART than in those who suffer a miscarriage. Hum Reprod. 2007;22(4):1134-1141. doi:10.1093/humrep/del458

28. Chien LW, Au HK, Chen PL, Xiao J, Tzeng CR. Assessment of uterine receptivity by the endometrial-subendometrial blood flow distribution pattern in women undergoing in vitro fertilization-embryo transfer. Fertil Steril. 2002;78(2):245-251. doi:10.1016/s0015-0282(02)032235

29. El Garhy IT, Mohamed AH,Sultan AS. Uterine and subendometrial arteries Doppler in patients with recurrent first trimestric abortion. Egypt J Hosp Med. 2018;73(5):6683-6690. doi:10.21608/ejhm.2018.16013

30. Adibi A, Khadem M, Mardanian F, Hovsepian S. Uterine and arcuate arteries blood flow for predicting of ongoing pregnancy in in vitro fertilization. J Res Med Sci. 2015;20(9):879-884. doi:10.4103/17351995.170622

31. Yalti S, Gürbüz B, Ficicioglu C, Canova H. Doppler evaluation of the uterine, intraovarian, stromal and spiral arteries on the day of human chorionic gonadotrophin administration in controlled ovarian hyperstimulation. J Obstet Gynaecol. 2003;23(4):402-406. doi:10.108 0/0144361031000120914

32. Elewa AM, Mansour AE, Gehad MA, Afify HE. Ovarian reserve testing and uterine blood flow assessment using two-dimensional and three-dimensional Doppler in patients with unexplained recurrent miscarriage. Benha Med J. 2017;34(2):81-87. doi:10.4103/bmfj. bmfj_107_17

33. Coulam CB, Goodman C, Rinehart JS. Colour Doppler indices of follicular blood flow as predictors of pregnancy after in-vitro fertilization and embryo transfer. Hum Reprod. 1999;14(8):1979-1982. doi:10.1093/humrep/14.8.1979

34. Elsandabesee D, Srinivas M, Kodakkattil S. The clinical value of combining maternal serum screening and uterine artery Doppler in prediction of adverse pregnancy outcome. J Obstet Gynaecol. 2006;26(2):115-117. doi:10.1080/01443610500443279

35. Schild RL, Holthaus S, d'Alquen J, et al. Quantitative assessment of subendometrial blood flow by three-dimensional-ultrasound is an important predictive factor of implantation in an in-vitro fertilization programme. Hum Reprod. 2000;15(1):89-94. doi:10.1093/ humrep/15.1.89

C 2021 The Author(s); This is an open-access article distributed under the terms of the Creative Commons Attribution License (http:// creativecommons.org/licenses/by/4.0), which permits unrestricted use, distribution, and reproduction in any medium, provided the original work is properly cited. 